\title{
Familial correlations in the Québec family study: cross-trait familial resemblance for body fat with plasma glucose and insulin
}

\author{
T. Rice ${ }^{1}$, A. Nadeau ${ }^{2}$, L. Pérusse ${ }^{3}$, C. Bouchard ${ }^{3}$, D. C. Rao ${ }^{1,4}$ \\ ${ }^{1}$ Division of Biostatistics, Washington University School of Medicine, St. Louis, Missouri, USA \\ ${ }^{2}$ Diabetes Research Unit, Laval University, Ste-Foy, Québec, Canada \\ ${ }^{3}$ Physical Activity Sciences Laboratory, PEPS, Laval University, Ste-Foy, Québec, Canada \\ ${ }^{4}$ Departments of Psychiatry and Genetics, Washington University School of Medicine, St. Louis, Missouri, USA
}

Summary This study represents one component in our investigation of the familial factors underlying the insulin resistance (or metabolic) syndrome involving obesity, hyperinsulinaemia, glucose intolerance, dyslipidaemia, and hypertension. Here we examine the cross-trait familial resemblance between four measures of body size (two assessing total fat [body mass index and sum of six skinfolds] and two assessing fat patterning [ratio of trunk skinfold sum to extremity skinfold sum, adjusted and unadjusted for total subcutaneous fat]) with fasting plasma levels of glucose, insulin, and the ratio of insulin to glucose (IGR) in non-diabetic families participating in phase 1 of the Québec Family Study. A bivariate familial correlation model assessed both intraindividual (e.g. father's body size with father's insulin) and interindividual (e.g. father's body size with son's insulin) cross-trait associations. Intraindividual correlations suggested a greater degree of cross-trait associations for body fat (rather than fat distribution) measures with insulin and the IGR (rather than with glucose) levels. While the intraindividual correlations were significant for most cross-trait comparisons, only the sum of six skinfolds evidenced any familial association (i.e. interindividual resemblance) with insulin and the IGR. Specifically, cross-trait parent-offspring (but not sibling or spouse) correlations were significant, with a bivariate familiality estimate (i.e. polygenic and/or common familial environment) of about $8 \%$. While the lack of sibling correlations does not suggest a simple familial hypothesis, a more complex genetic effect underlying the common covariation between total body fat with insulin and IGR cannot be ruled out. [Diabetologia (1996) 39: 1357-1364]

Keywords Bivariate, genetic, environmental, pleiotropy.
Upper body and abdominal obesity are associated with a cluster of metabolic conditions which can lead to increased risk for cardiovascular disease, stroke, and non-insulin-dependent diabetes mellitus (NIDDM). This so-called metabolic syndrome (or syndrome X) includes insulin resistance, hyperinsulinaemia, impaired glucose tolerance, dyslipidaemia,

Received: 4 August 1995 and in final revised form: 7 June 1996

Corresponding author: Dr. T. Rice, Washington University School of Medicine, Division of Biostatistics, Box 8067, 660 S. Euclid Ave., St. Louis, MO 63110, USA

Abbreviations: IGR, Insulin-to-glucose ratio; BMI, body mass index; SF6, sum of six skinfolds; TER, trunk-to-extremity skinfold ratio; TER-sf, TER adjusted for SF6; NIDDM, non-insulin-dependent diabetes mellitus; QFS, Québec Family Study. hypertension, and a variety of other metabolic derangements $[1,2]$, and is generally observed in the presence of an obese state. Insulin resistance, with compensatory hyperinsulinaemia, may be the central element in understanding the basis of this metabolic cluster.

Insulin resistance and hyperinsulinaemia are commonly observed in obese people, particularly those with upper body and visceral obesity [3, 4]. In contrast, weight loss is associated with a decrease in insulin resistance [5] while weight gain is accompanied by reduced insulin sensitivity [6,7]. Furthermore, adjusting insulin level for the effects of obesity and fat distribution accounts for its ability to predict later NIDDM [8]; that is, obesity and insulin share the same predictive power. 
The genetic and non-genetic bases for these associations are still unclear. Common metabolic paths imply genetic pleiotropy (i.e. one or more genes influencing both traits). However, the covariation between body fat and insulin levels suggests there may be environmental influences, as well as gene-by-environment interactions. For example, nutrition and exercise may influence both weight and insulin levels in a similar way, depending on genotype.

Most of the evidence for a genetic basis for the metabolic syndrome is based on univariate studies (i.e. a single trait at a time) as recently reviewed [9]. For example, a (putative) major gene for hyperinsulinaemia was found in normoglycaemic relatives of NIDDM patients [10]. Those individuals with the recessive genotype (hyperinsulinaemic) were also more obese, and had increased dyslipidaemia and hypertension as compared to those without the recessive genotype. Genetic linkage, correlated genetic and environmental effects, and gene by environment interactions could all explain this pattern of results. In fact, both genetic and common environmental effects have been shown to account for the covariation between body fat and blood pressure as also predicted by the metabolic syndrome $[11,12]$.

Given the recent interest in the metabolic syndrome, which contends that the various traits (especially insulin levels and obesity) share a common physiological basis (evidenced by clustering at the individual phenotypic level), the essential question to geneticists is whether or not, and to what extent, the measures share a common genetic basis. Whether the underlying factors causing variation in a trait (or pairs of traits) are heritable or not can be distinguished using genetic epidemiology methods. An inspection of the significant familial patterns can lead to certain genetic and environmental inferences. For example, a pattern of significant correlations among siblings and between parents and offspring (who share about half their genes), but not between spouses (who share few genes assuming random mating), suggests a genetic heritability. Significant spouse correlations, in addition to sibling and parent-offspring, suggest that at least some of the familial effect may be due to shared environments. Similarly, cross-trait familial correlations lead to the same type of genetic and environmental inferences. A pattern of significant cross-trait correlations between parents' body size and offspring's insulin level, and among siblings, but not between spouses, would suggest that a common gene (or genes) influences both traits. On the other hand, significant cross-trait correlations within individuals, but not between family members, suggests non-familial causes for the covariation between traits.

The present study is one component of a more complete investigation of the factors which underlie various traits in the metabolic syndrome [12]. Here, data from the Québec Family Study (QFS) is used to examine the cross-trait familial resemblance between fasting plasma glucose and insulin levels with several measures of body fat.

\section{Subjects and methods}

Sample. The QFS consists of families of French descent living within $80 \mathrm{~km}$ around Québec City who were recruited through the media during the years 1978-1981 in order to study the genetic and environmental effects on several physiological and biochemical traits. A total of 1630 non-diabetic individuals, comprising 375 families, were ascertained. Individuals in the parent generation $(n=727)$ range in age from 30.2 to 59.5 years, while the offspring $(n=903)$ range from 8.4 to 25.7 years. The average $( \pm \mathrm{SD})$ socioeconomic status rating [13] of the fathers is slightly above $(54.1 \pm 14.19$, ranging from 23.0-75.3) that of the general French-Canadian population [14].

The sample includes nuclear families consisting of parents and singleton offspring, as well as families with twin and/or adopted offspring, step-parents, or cousins. For the purposes of age and sex adjustments (described below), the complete sample was used. However, for the familial correlation analyses, only traditional nuclear families consisting of parents and their singleton biological offspring were retained. Plasma samples were not available for all subjects, leading to reduced sample sizes for the insulin measures. Sample sizes used in the familial analyses are given later.

Diabetic subjects and their families were not included in this study. A two-stage procedure was used in order to ensure this. In a medical examination prior to the study, the family was removed from the project if fasting glucose levels revealed a diabetic state. Further tests were conducted to confirm the preliminary diagnosis made on the basis of fasting glucose levels for the benefit of the excluded families.

Measures. A wide variety of physiological and behavioural measurements was obtained during a 1-day visit of the families to the laboratory. Measures relating to body fat include height $(\mathrm{m})$, weight $(\mathrm{kg})$, and six skinfold thicknesses $(\mathrm{mm})$. The body mass index (BMI) was computed with weight and height $(\mathrm{kg} /$ $\mathrm{m}^{2}$ ). Six measures of skinfold thicknesses (suprailiac, subscapular, abdominal, medial calf, biceps, triceps) were obtained on the left side of the body with a Harpenden skinfold caliper as recommended by the International Biological Programme [17]. Further details regarding measurement and reliability of the skinfolds have been described previously $[15,16]$.

Two variables were extracted from the six skinfolds, the sum of all six (SF6), and the trunk-to-extremity ratio (TER, [suprailiac + subscapular + abdominal $] /[$ medial calf + biceps + triceps]). The SF6 is a summary measure, representing the absolute amount of subcutaneous fat. The TER assesses the pattern of subcutaneous fat distribution, with high scores indicating greater truncal than extremity fat deposition. The TER was also adjusted for total subcutaneous fat (SF6) by regression analysis (TER-sf). The TER-sf indexes the preferential deposition of fat in the upper body or the lower body independently of the amount of subcutaneous fat. The regression analysis for TER-sf [12] consisted of a stepwise procedure, extracting the standardized residual from the regression of TER on up to a cubic polynomial in SF6. SF6 effects accounted for between $11.7 \%$ (daughters) and $19.1 \%$ (fathers) of the variance in TER. 
Table 1. Sample statistics for raw variables, by sex and generation groups ${ }^{\mathrm{a}}$

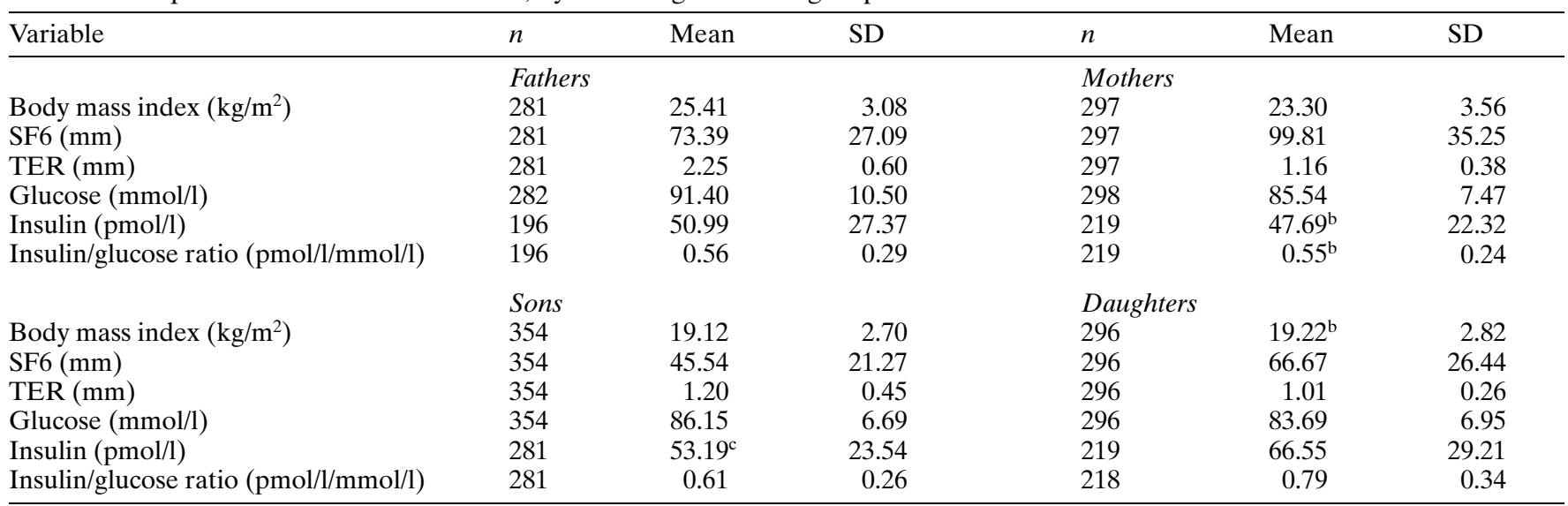

${ }^{a}$ Cross-trait sample size includes 307 families (307 spouse pairs, 1300 parent-offspring pairs, and 438 sibling pairs), with sibship size ranging from 1 through 4 . ${ }^{\mathrm{b}}$ Non-significant sex (within generation) difference, based on a comparison of standard errors. ${ }^{c}$ Non-significant generation (within sex) difference, based on a comparison of standard errors
Plasma glucose (mmol/l) was assessed enzymatically using standard procedures [18]. Serum insulin (pmol/l) was determined by radioimmunoassay [19]. In addition to glucose and insulin, the ratio of insulin/glucose (IGR) was also analysed. Table 1 gives the means and standard deviations of the unadjusted variables, separately in four sex-by-generation groups. Based on a comparison of standard errors, there were generation differences for all variables except for insulin in males. There was a general trend for higher values in parents than in offspring, except for insulin and IGR. There were also sex differences for all variables, except for BMI in offspring and insulin and IGR in parents. The sample statistics in Table 1 represent the sub-sample used in the familial correlation analyses.

Data adjustments. Each of the body size and plasma variables were adjusted for the effects of age, separately in four sex by generation groups since mean differences were previously noted. In summary, extreme outliers (beyond mean \pm 4 SD) were temporarily set aside so that the regression models would not be unduly influenced by extreme observations. A given measure was regressed on up to a cubic polynomial in age in a stepwise manner, retaining terms significant at the $5 \%$ level. The residual variance was also examined for age effects (heteroscedasticity) by regressing the squared residual from the above age regression (or the log of the squared residual) on another polynomial in age in a stepwise manner and retaining terms significant at the $5 \%$ level. The final phenotypes were computed for all individuals (including the extreme observations) by using the best regression models, or by standardizing to zero mean and unit variance within sex and generation groups if no age terms were significant.

Data adjustments for glucose included mean age effects in fathers (linear term accounting for $2.25 \%$ of the variance) and daughters (age $\mathrm{ar}^{3}$ term accounting for $3.37 \%$ of the variance). No mean age effects were noted in the mothers or sons, nor was there any heteroscedasticity. No age effects were found for insulin, or for IGR.

Age regressions for the four body fat measures are to be found elsewhere [12, 20, 21]. In general, $5 \%$ or less of the variance was accounted for by age, with a few exceptions. A full cubic polynomial in age accounted for 47 and $50 \%$ of the variance in sons TER and BMI (respectively), and 10 and $26 \%$ in daughters (respectively).
Intraindividual correlations between SF6 and BMI were moderately high, ranging from about 0.6 to 0.8 . High correlations were also noted between TER and TER-sf (0.87-0.94). Both are included since one (TER) assesses the overall pattern of fat distribution while the other also takes into consideration the total level of fatness. In general, the correlations of TER and TER-sf with SF6 and BMI were low to non-significant.

Familial correlations. The familial correlation model may be conceptualized as a simple extension of the univariate case involving four types of individuals (fathers $[\mathrm{F}]$, mothers $[\mathrm{M}]$, sons [S], and daughters [D]), leading to eight interindividual correlations within three familial classes (one spouse [FM], four parent-offspring [FS, FD, MS, and MD], and three sibling $[\mathrm{SS}, \mathrm{DD}, \mathrm{SD}])$. In expanding to the bivariate case, each of the eight correlations becomes a matrix of correlations (see Appendix 1). Finally, there are four additional correlations representing the intraindividual cross-trait resemblance within each of the fathers, mothers, sons, and daughters. Although there are a total of 32 correlations in this bivariate model, only 18 of them involve cross-trait resemblance (e.g. body fat in fathers with insulin in offspring - see Appendix 1), which is the primary interest in this study. We did not test the significance of each of the 18 correlations individually, but rather tested the familial patterns by familial class (e.g. spouse or sibling), as explained more fully later. The finding of significant crosstrait familial correlations was consistent with the hypothesis that the same familial factors (i.e. genes and/or common environments) influence both traits. Furthermore, if the familial cross-trait effect was due in part to genetic factors, then the finding was also consistent with an hypothesis of pleiotropy (i.e. the same gene(s) influences both traits).

The cross-trait correlations are expressed using element notation. For example, the spouse correlation $\mathrm{f}_{1} \mathrm{~m}_{2}\left(\right.$ and $\left.\mathrm{f}_{2} \mathrm{~m}_{1}\right)$ represents body fat in fathers with insulin in mothers (and vice versa) and the parent-offspring correlation $\mathrm{f}_{1} \mathrm{~s}_{2}\left(\right.$ and $\left.\mathrm{f}_{2} \mathrm{~s}_{1}\right)$ represents body fat in fathers with insulin in sons (and vice versa). Similarly, the sibling correlation $\mathrm{s}_{1} \mathrm{~d}_{2}$ (and $\mathrm{s}_{2} \mathrm{~d}_{1}$ ) represents body fat in sons with insulin in daughters (and vice versa). Finally, the intraindividual correlation $\mathrm{f}_{12}$ represents the crosstrait resemblance in fathers. The computer program SEGPATH [22] was used to estimate the familial correlations by maximum likelihood methods. The method for the particular correlation model used here has been more fully outlined 
previously [12]. Hypotheses were tested using the likelihood ratio, which is the difference in (minus twice) the log-likelihoods obtained under the two nested models. The likelihood ratio is distributed as a chi-square, with the degrees of freedom $(d f)$ being the difference in the number of parameters estimated in the two competing hypotheses.

Competing hypotheses were tested by comparing the likelihood from the general model (all 34 correlations estimated), to that from a reduced model. A series of reduced models was estimated in order to test the correlations by familial class (such as sibling, parent-offspring, or spouse) for significance and for certain sex-specific and cross-trait hypotheses. The main question addressed here is whether there is cross-trait resemblance by familial class. For example, the sibling cross-trait correlations were tested for significance by fixing them to zero $\left(\mathrm{s}_{1} \mathrm{~s}_{2}=\mathrm{d}_{1} \mathrm{~d}_{2}=\mathrm{s}_{1} \mathrm{~d}_{2}=\mathrm{s}_{2} \mathrm{~d}_{1}=0\right)$ and comparing that likelihood to the general model, with $4 d f$. The significance of the parent-offspring $\left(\mathrm{f}_{1} \mathrm{~s}_{2}=\mathrm{f}_{2} \mathrm{~s}_{1}=\mathrm{f}_{1} \mathrm{~d}_{2}=\mathrm{f}_{2} \mathrm{~d}_{1}=\mathrm{m}_{1} \mathrm{~s}_{2}=\mathrm{m}_{2} \mathrm{~s}_{1}=\mathrm{m}_{1} \mathrm{~d}_{2}=\mathrm{m}_{2} \mathrm{~d}_{1}=0\right.$, $d f=8)$, spouse $\left(\mathrm{f}_{1} \mathrm{~m}_{2}=\mathrm{f}_{2} \mathrm{~m}_{1}=0, d f=2\right)$, and intraindividual $\left(\mathrm{f}_{12}=\mathrm{m}_{12}=\mathrm{s}_{12}=\mathrm{d}_{12}=0, d f=4\right)$ cross-trait correlations were similarly tested by familial class. Offspring sex differences were tested separately in the parent-offspring (i.e., $\mathrm{f}_{1} \mathrm{~s}_{2}=\mathrm{f}_{1} \mathrm{~d}_{2}$, $\left.\mathrm{f}_{2} \mathrm{~s}_{1}=\mathrm{f}_{2} \mathrm{~d}_{1}, \quad \mathrm{~m}_{1} \mathrm{~s}_{2}=\mathrm{m}_{1} \mathrm{~d}_{2}, \quad \mathrm{~m}_{2} \mathrm{~s}_{1}=\mathrm{m}_{2} \mathrm{~d}_{1}, \quad d f=4\right)$ and sibling $\left(\mathrm{s}_{1} \mathrm{~s}_{2}=\mathrm{d}_{1} \mathrm{~d}_{2}=\mathrm{s}_{1} \mathrm{~d}_{2}=\mathrm{s}_{2} \mathrm{~d}_{1}, d f=3\right)$ familial classes, as were sex differences in both parents and offspring in the parent-offspring familial class $\left(\mathrm{f}_{1} \mathrm{~s}_{2}=\mathrm{f}_{1} \mathrm{~d}_{2}=\mathrm{f}_{2} \mathrm{~s}_{1}=\mathrm{f}_{2} \mathrm{~d}_{1}=\mathrm{m}_{1} \mathrm{~s}_{2}=\mathrm{m}_{1} \mathrm{~d}_{2}=\mathrm{m}_{2} \mathrm{~s}_{1}=\right.$ $\left.\mathrm{m}_{2} \mathrm{~d}_{1}, d f=7\right)$. No sex or generation differences in the cross-trait correlations were also tested $\left(\mathrm{f}_{1} \mathrm{~s}_{2}=\mathrm{f}_{1} \mathrm{~d}_{2}=\mathrm{f}_{2} \mathrm{~s}_{1}=\mathrm{f}_{2} \mathrm{~d}_{1}=\mathrm{m}_{1} \mathrm{~s}_{2}=\right.$ $\left.\mathrm{m}_{1} \mathrm{~d}_{2}=\mathrm{m}_{2} \mathrm{~s}_{1}=\mathrm{m}_{2} \mathrm{~d}_{1}=\mathrm{s}_{1} \mathrm{~s}_{2}=\mathrm{d}_{1} \mathrm{~d}_{2}=\mathrm{s}_{1} \mathrm{~d}_{2}=\mathrm{s}_{2} \mathrm{~d}_{1}, \quad d f=11\right)$. The most parsimonious model incorporates all the nonrejected hypotheses from all of the sex, generation, and cross-trait tests.

\section{Results}

Table 2 is a summary of the most parsimonious models of cross-trait resemblance for each of the pairs of measures. Cross-trait correlations are noted separately for intraindividual compared to interindividual comparisons. "Yes" signifies that the correlations are significant $(p<0.05)$, "No" denotes non-significance, and "Some" reflects that at least some of the correlations (spouse or parent-offspring or sibling) were significant or borderline. As shown in Table 2, none of the cross-trait correlations (either intraindividual or interindividual) involving the TER-sf were significant. Although the intraindividual correlations involving the BMI and TER were significant, none of the interindividual correlations were. For SF6, both intraindividual and some interindividual cross-trait resemblance is noted with the insulin and IGR measures. As noted in Table 2, the parent-offspring correlations were significant, while the spouse and sibling correlations were not.

Intraindividual cross-trait correlations $( \pm S E)$ are given in Table 3 . The general pattern noted here is that glucose, insulin and IGR were more strongly associated with body fat (BMI and SF6) than with fat distribution measures (TER, TER-sf). Furthermore, the body size measures were more strongly correlated with insulin and IGR than with glucose. There also appear to be generation differences in the insulin
Table 2. Summary of cross-trait resemblance ${ }^{\mathrm{a}}$

\begin{tabular}{llllll}
\hline & & BMI & SF6 & TER-sf & TER \\
\hline Glucose & Intraindividual & Yes & Yes & No & Yes \\
& Interindividual & No & No & No & No \\
Insulin & Intraindividual & Yes & Yes & No & Yes \\
& Interindividual & No & Some $^{\text {b }}$ & No & No \\
Insulin/ & Intraindividual & Yes & Yes & No & Yes \\
glucose ratio & Interindividual & No & Some $^{c}$ & No & No \\
\hline
\end{tabular}

${ }^{a}$ Cross-trait resemblance is separately defined for intraindividual and interindividual correlations: "Yes" designates significant $(p<0.05)$ correlations; "No" denotes non-significance; and "Some" signifies that at least some of the correlations are significant. ${ }^{\mathrm{b}}$ Cross-trait correlations for parent-offspring are significant $(p=0.025)$, and suggestive for spouses $(p=0.087)$. ${ }^{c}$ Cross-trait correlations for parent-offspring are significant $(p=0.025)$, while the spouse $(p=0.103)$ and sibling correlations $(p=0.155)$ are suggestive

Table 3. Maximum likelihood estimates of intraindividual cross-trait correlations ( \pm standard errors) under the most parsimonious models ${ }^{\mathrm{a}}$

\begin{tabular}{lllll}
\hline Measure & BMI & SF6 & TER-sf $^{\mathrm{b}}$ & TER \\
\hline Glucose & & & & \\
$\mathrm{f}_{12}$ & $0.16 \pm 0.02$ & $0.17 \pm 0.02$ & $0.04 \pm 0.03$ & $0.08 \pm 0.02$ \\
$\mathrm{~m}_{12}$ & {$[0.16]$} & {$[0.17]$} & {$[0.04]$} & {$[0.08]$} \\
$\mathrm{s}_{12}$ & {$[0.16]$} & {$[0.17]$} & {$[0.04]$} & {$[0.08]$} \\
$\mathrm{d}_{12}$ & {$[0.16]$} & {$[0.17]$} & {$[0.04]$} & {$[0.08]$} \\
Insulin & & & & \\
$\mathrm{f}_{12}$ & $0.43 \pm 0.05$ & $0.44 \pm 0.05$ & $0.07 \pm 0.07$ & $0.18 \pm 0.06$ \\
$\mathrm{~m}_{12}$ & $0.39 \pm 0.05$ & $0.45 \pm 0.05$ & $0.09 \pm 0.07$ & $0.19 \pm 0.05$ \\
$\mathrm{~s}_{12}$ & $0.21 \pm 0.05$ & $0.28 \pm 0.05$ & $0.04 \pm 0.07$ & $0.08 \pm 0.05$ \\
$\mathrm{~d}_{12}$ & $0.15 \pm 0.06$ & $0.20 \pm 0.06$ & $0.08 \pm 0.07$ & $0.05 \pm 0.05$ \\
Insulin/glucose ratio & & & \\
$\mathrm{f}_{12}$ & $0.43 \pm 0.05$ & $0.42 \pm 0.05$ & $0.07 \pm 0.07$ & $0.17 \pm 0.06$ \\
$\mathrm{~m}_{12}$ & $0.36 \pm 0.05$ & $0.41 \pm 0.05$ & $0.08 \pm 0.07$ & $0.16 \pm 0.05$ \\
$\mathrm{~s}_{12}$ & $0.20 \pm 0.05$ & $0.25 \pm 0.05$ & $0.02 \pm 0.07$ & $0.07 \pm 0.05$ \\
$\mathrm{~d}_{12}$ & $0.14 \pm 0.06$ & $0.20 \pm 0.06$ & $0.10 \pm 0.07$ & $0.05 \pm 0.05$ \\
\hline
\end{tabular}

a Significant sex and generation effects were noted for insulin and IGR (respectively) with BMI $(p=0.008,0.008)$, SF6 $(p=0.014,0.012)$, TER-sf $(p=0.030,0.027)$, and TER $(p=0.028,0.027)$. No sex or generation differences were noted for any glucose analyses. ${ }^{\mathrm{b}}$ None of the TER-sf intraindividual correlations are significant based on likelihood ratios

and IGR correlations, with higher cross-trait resemblance in the older (parent) than younger (offspring) group.

The maximum likelihood estimates of the interindividual cross-trait correlations for SF6 with insulin and IGR are given in Table 4, under both the general and most parsimonious models. Although the sibling correlations were not significant when tested by familial class, it is noteworthy that the individual daughter-daughter correlations were quite high in the general model. The largest parent-offspring correlations also involve daughters $\left(\mathrm{f}_{2} \mathrm{~d}_{1}\right.$ and $\left.\mathrm{m}_{1} \mathrm{~d}_{2}\right)$. The bivariate familiality (i.e. polygenic and/or common environmental effects), which was obtained by simply doubling the average familial correlation, was about $8 \%$. 
Table 4. Maximum likelihood estimates of interindividual cross-trait correlations ( \pm standard errors)

\begin{tabular}{|c|c|c|c|c|}
\hline \multirow[t]{2}{*}{ Parameters } & \multicolumn{2}{|c|}{ SF6 and insulin } & \multicolumn{2}{|l|}{$\underline{\text { SF6 and IGR }}$} \\
\hline & General & Parsimonious & General & Parsimonious \\
\hline \multicolumn{5}{|l|}{ Spouse } \\
\hline $\mathrm{f}_{2} \mathrm{~m}_{1}$ & $-0.06 \pm 0.07$ & {$[0]$} & $-0.06 \pm 0.07$ & [0] \\
\hline \multicolumn{5}{|c|}{ Parent-offspring } \\
\hline $\mathrm{f}_{1} \mathrm{~s}_{2}$ & $0.03 \pm 0.06$ & $0.05 \pm 0.06$ & $0.03 \pm 0.06$ & $0.05 \pm 0.06$ \\
\hline $\mathrm{f}_{2} \mathrm{~d}_{1}$ & $0.19 \pm 0.08$ & $0.14 \pm 0.08$ & $0.20 \pm 0.08$ & $0.15 \pm 0.08$ \\
\hline $\mathrm{m}_{1} \mathrm{~s}_{2}$ & $0.02 \pm 0.06$ & $0.02 \pm 0.06$ & $0.01 \pm 0.06$ & $0.01 \pm 0.06$ \\
\hline $\mathrm{m}_{2} \mathrm{~s}_{1}$ & $-0.00 \pm 0.06$ & $0.03 \pm 0.05$ & $-0.01 \pm 0.06$ & $0.02 \pm 0.05$ \\
\hline $\mathrm{m}_{1} \mathrm{~d}_{2}$ & $0.22 \pm 0.06$ & $0.20 \pm 0.06$ & $0.21 \pm 0.06$ & $0.19 \pm 0.06$ \\
\hline $\mathrm{m}_{2} \mathrm{~d}_{1}$ & $0.10 \pm 0.07$ & $0.07 \pm 0.07$ & $0.12 \pm 0.07$ & $0.08 \pm 0.07$ \\
\hline \multicolumn{5}{|l|}{ Sibling } \\
\hline
\end{tabular}

Subscript 1 , body fat; subscript 2 , insulin or IGR

\section{Discussion}

Cross-trait familial resemblance between fasting plasma glucose and insulin levels with several measures of total fat and fat distribution was examined using a bivariate familial correlation model. The bivariate familial model as used here is a general exploratory and screening method. Its primary purpose is to isolate, from a larger set of variables, pairs of measures which suggest common aetiological determinants. Familial correlations underlie most complex models in genetic epidemiology, involve few assumptions, and are efficient for screening a large number of comparisons. More complex genetic models such as bivariate segregation and linkage studies, while providing more definitive answers to these questions, involve a significant cost in terms of restrictive assumptions and time. Therefore, preliminary studies such as this serve a useful function in delineating specific hypotheses for more detailed examinations.

Familial cross-trait resemblance was observed only for insulin and IGR with total fat; although fat distribution was associated with insulin within individuals, no evidence for a familial cross-trait component was found. This result emphasizes that caution is warranted when inferring familial causes (either genetic or common environmental) for associations based on population or intraindividual studies. Traits may be correlated because of specific factors which are unique to each individual, and thus are not shared among family members and are not heritable.

The null results arising from this study are quite revealing as regards the specific body size attributes which relate to the insulin resistance syndrome - i.e. TER (a measure of fat distribution) shared no common familial aetiologies with insulin, nor did TER- sf. The TER-sf is the residual resulting from the regression of total subcutaneous fat on the TER, and it represents the fat distribution pattern independent of total level of subcutaneous fat. Therefore, removal of the variance in TER due to amount of subcutaneous fat leads to no remaining covariation with insulin or the IGR. This result confirms that the specific body size attribute is total fat rather than fat distribution patterns.

Recent research in population studies stress that the most relevant body size component related to the metabolic syndrome is intra-abdominal fat [2]. That is, within individuals, abdominal visceral obesity is associated with multiple endocrine abnormalities. Unfortunately, measures of visceral obesity were not assessed in phase 1 of the QFS. However, this component was implicated in other analyses not reported in detail, including percent body fat (\% body fat measured with underwater weighing), and the skinfold sum adjusted for total fat mass (which was derived from $\%$ body fat and weight) $[15,16]$. The ratio (SF6/total fat mass) represents the percentage of total fat which is subcutaneous. Analyses not reported in detail suggested that insulin and IGR were crosscorrelated (significant intra- and interindividual) with total fat (\% body fat results were similar to those for SF6), but not for SF6/total fat mass. These findings implicate internal fat stores. Since insulin and IGR are related to both total fat (\% body fat) and total subcutaneous fat (SF6), but not to the percentage which is subcutaneous (SF6/total fat mass), an indirect relationship is implied with nonsubcutaneous (or internal) fat. Thus, the QFS indirectly verifies that the previously noted visceral obesity/insulin association may in fact be due to familial (perhaps genetic) sources. Direct measures of internal fat (specifically 
abdominal visceral fat) are needed in order to address this particular aspect of the metabolic syndrome.

The novel finding in this study regards the relationship between SF6 (total subcutaneous fat) with insulin and IGR. The pattern of non-significant sibling correlations for insulin with SF6 was surprising given the familial association noted in parents and offspring. However, the SF6-IGR sibling correlations were suggestive $(p=0.155)$ given the overall pattern of results (i.e. sex differences in the siblings and significant parent-offspring correlations). In addition, although the sibling correlations were not significant as a group, individual correlations were significant (e.g. daughter-daughter pairs and cross-sex pairs involving daughter's body fat and son's insulin or IGR) based on a comparison of standard errors. Thus, while a simple pleiotropy hypothesis is not strictly supported for these pairs of measures, it also cannot be clearly ruled out.

Together, these results suggest that the pleiotropic hypothesis is likely to be influenced by both sex and maturational status. Similar to the sex effect seen in the sibling correlations, there was also a sex of offspring effect in the parent-offspring correlations. Specifically, the average parent-daughter correlation (0.11) was more than twice as large as the average parent-son correlation (0.03). Thus, given that there is evidence for sex differences in the offspring involving both sibling and parent-offspring comparisons, the possibility of a sex-dependent familial effect is likely. Possible explanations for the discrepancies in the correlations between the sons and daughters may be related to several factors such as age or sexual maturation differences, body fat differences, and other factors affecting insulin sensitivity such as physical activity levels which may differ between the sexes.

Regarding maturational effects, it is well known that differences in body composition and insulin levels emerge during puberty, which also vary by sex. These differences may persist even after age adjustments (conducted separately by sex), especially if the effects are in part a function of familial factors. Therefore, inclusion of both pre- and post-pubertal offspring in these analyses may have affected the results, and may be the primary cause of the non-significant sibling correlations. However, analysis of these data separately by pre- and post-pubertal offspring was not possible due to the small sample sizes (and reduced power) which result (nearly $60 \%$ of the offspring are pre-pubertal).

Maturational-by-sex differences may also relate to a pattern noted in the parent-offspring correlations involving the daughters, where the correlation between father's insulin and daughter's fat $\left(\mathrm{f}_{2} \mathrm{~d}_{1}\right)$ apparently was more similar than the reverse $\left(\mathrm{f}_{1} \mathrm{~d}_{2}\right)$; the opposite pattern was noted for mothers and daughters $\left(\mathrm{m}_{1} \mathrm{~d}_{2}>\mathrm{m}_{2} \mathrm{~d}_{1}\right)$. A simple pleiotropic hypothesis would predict that these cross-trait comparisons should be equal (i.e. $\mathrm{f}_{2} \mathrm{~d}_{1}=\mathrm{f}_{1} \mathrm{~d}_{2}$ and $\mathrm{m}_{1} \mathrm{~d}_{2}=\mathrm{m}_{2} \mathrm{~d}_{1}$ ), and in fact a comparison of the standard errors associated with each estimate suggests that they may not be discrepant. Whether the pleiotropic hypothesis is affected by maturational and/or sex status can be addressed more precisely in a later phase of the QFS in which all individuals will be measured approximately 12 years after the original study, thus ensuring that nearly all of the offspring are post-pubertal.

It is also noteworthy that the test for spouse crosstrait correlations for SF6 was suggestive ( $p=0.08$ to $0.10)$ with insulin and IGR. Examination of the magnitude of those correlations again suggested sex differences, with insulin in mothers and SF6 in fathers being more highly correlated than the reverse. Spouse correlations suggest that there may well be familial environmental effects, and the pattern suggests they may be dependent on sex. Furthermore, the larger parent than offspring intraindividual correlations suggest a generation (or age) effect in the cross-trait resemblance (perhaps attributed to maturational effects in the offspring). Moreover, the higher intrathan inter- individual correlations suggests that there are other (non-familial) causes for the covariation between SF6 with insulin and IGR.

A recent study [23] investigating the familial relationship between insulin levels (both fasting and 2-h post-glucose load) and several insulin-resistance syndrome traits (including BMI, subscapular/triceps skinfold ratio, waist/hip ratio) in families where all individuals were post-pubertal, suggested that also BMI shared genetic and environmental aetiologies with insulin. In contrast, a recent twin study [24] investigating the relationship between insulin and BMI suggested that the association appeared to occur independently of genetic influences, and were largely explained by non-genetic (behavioural or environmental) factors. While the results from the current study also suggest that the covariation between insulin and BMI are significant at the phenotypic level, there was no evidence of shared familial aetiologies (i. e. significant intraindividual but not interindividual cross-trait resemblance). Since the BMI is a complex phenotype, consisting of both fat and lean mass, as well as bone tissue, there may well be several genetic and/or environmental aetiologies. A review of segregation studies of the BMI suggest that there are as many negative results (i.e. no support for a major gene [25-28] as there are positive results [29-32]. Also, although most of these studies report an autosomal recessive form of transmission [29-32], at least one study suggests that there may be both a recessive locus and a co-dominant locus, depending on how the data are subsetted [33]. Thus, assuming that there are several major influences on the BMI, it is not surprising that different results are found across different 
samples in which different alleles and/or loci may dominate in the expression of obesity.

There are at least three major findings in this study. First, there may be more than one genetic factor underlying this syndrome. According to some definitions of the metabolic syndrome $[1,2,9]$, we expected insulin to show the strongest relationship with TER, assuming it is a proxy for upper body fat. That expectation was not met here, although the fat distribution measure did have a familial association with blood pressure in these data [12]. Together, these combined studies suggest at least two underlying familial factors relating obesity to the other metabolic syndrome traits-one involving total fat and insulin levels, and another involving blood pressure and fat distribution or upper body fat. This hypothesis is also indirectly supported in a recent study [23], where fasting and 2-h post-glucose load insulin levels shared common genetic aetiologies with a proxy measure of total fat (as assessed with the BMI), but not with systolic or diastolic blood pressure. Second, the finding that insulin was related to total fat (SF6 and $\%$ body fat), but not to the percentage which was subcutaneous, indirectly implicates a familial association between insulin levels and internal (or abdominal visceral) fat. Third, the familial relationship between insulin and total body fat (with a bivariate familiality estimate of about $8 \%$ ) may involve complex interactions with sex and/or age, maturational status, and "environmental" factors such as activity levels. Therefore, caution is warranted in interpreting these results. The $8 \%$ bivariate heritability between insulin and SF6 may well be an underestimate of the "true" value, given the possible confounding of maturational effects and sex differences. Given that these analyses cannot rule out the possibility of shared physiological (or genetic) aetiologies for both insulin levels and total fat, more complex genetic epidemiological research is needed involving bivariate segregation analysis with genotype-dependent effects of co-variates such as age, sex and physical activity levels. Moreover studies incorporating "adult" offspring, along with measurements of abdominal visceral fat, glucose tolerance, and indicators of insulin sensitivity are needed in order to fully understand the physiological bases underlying the metabolic syndrome.

Acknowledgements. This work was partly supported by NIH grant GM-28719 and MRC of Canada grant PG-11811.

\section{References}

1. Reaven GM (1988) Role of insulin resistance in human disease. Diabetes 37: 1595-1607

2. Björntorp P (1992) Abdominal obesity and the metabolic syndrome. Ann Med 24: 465-468

3. Kissebah AH, Freedman DS, Peiris AN (1989) Health risks of obesity. Med Clin North Am 73: 111-138
4. Bouchard C, Després JP, Mauriège P (1993) Genetic and nongenetic determinants of regional fat distribution. Endocr Rev 14: 72-93

5. DeFronzo RA, Ferrannini E (1991) Insulin resistance: a multifaceted syndrome responsible for NIDDM, obesity, hypertension, dyslipidemia, and atherosclerotic cardiovascular disease. Diabetes Care 14: 173-194

6. Oppert JM, Vohl MC, Chagnon M et al. (1995) DNA polymorphism in the uncoupling protein (UCP) gene and human body fat. Int J Obesity 18: 526-531

7. Krotkiewski M, Mandroukas K, Sjöström L, Sullivan L, Wetterqvist H, Björntorp P (1979) Effects of long-term physical training on body fat, metabolism, and blood pressure in obesity. Metabolism 28: 650-658

8. Haffner SM, Valdez RA, Hazuda HP, Mitchell BD, Morales PA, Stern MP (1992) Prospective analysis of the insulin-resistance syndrome (syndrome X). Diabetes 41: 715722

9. Bouchard C, Pérusse L (1994) Genetics of causes and manifestations of the metabolic syndrome. In: Padova G, Crepaldi G (eds) 6th European Symposium on metabolism: the plurimetabolic syndrome.Elsevier Science Publishers, Amsterdam, pp 67-74

10. Schumacher MC, Hasstedt SJ, Hunt SC, Williams RR, Elbein SC (1992) Major gene effect for insulin levels in familial NIDDM pedigrees. Diabetes 41: 416-423

11. Schork NJ, Weder AB, Trevisan M, Laurenzi M (1994) The contribution of pleiotropy to blood pressure and body mass index variation: the Gubbio study. Am J Hum Genet 54: 361-373

12. Rice T, Province M, Pérusse L, Bouchard C, Rao DC (1994) Cross-trait familial resemblance for body fat and blood pressure: familial correlations in the Québec Family Study. Am J Hum Genet 55: 1019-1029

13. Blishen BR, McRoberts HA (1976) A revised index for occupations in Canada. Can Rev Soc Anthropol 13: 71-79

14. Blishen BR (1970) Social class and opportunity in Canada. Can Rev Soc Anthropol 7: 110-172

15. Himes JH, Bouchard C (1985) Do the new Metropolitan Life Insurance weight-height tables correctly assess body frame and body fat relationships? Am J Public Health 75: 1076-1079

16. Bouchard C (1985) Reproducibility of body-composition and adipose-tissue measurements in humans. In: AF Roche (ed) Body-composition assessments in youth and adults: report of the Sixth Ross Conference on medical research. Ross Laboratories, Columbus, Ohio, pp 9-14

17. Weiner JS, Lourie JA (1969) Human biology: a guide to field methods. Blackwell, Oxford

18. Richterich R, Dauwalder H (1971) Zur Bestimmug der Plasmaglucosekonzentra-tion mit der Hexokinase-glucose-6-phosphat-dehydrogenase-methode. Schweiz Med Wochenschr 101: 615-618

19. Desbuquois B, Aurbach GD (1971) Use of the polyethylene glycol to separate free and antibody-bound peptide hormones in radioimmunoassays. J Clin Endocrinol Metab 37: 732-738

20. Borecki IB, Rice T, Bouchard C, Rao DC (1991) Commingling analysis of generalized body mass and composition measures: the Québec Family Study. Int J Obesity 15: 763773

21. Rice T, Borecki IB, Bouchard C, Rao DC (1992) Commingling analysis of regional fat distribution measures: the Québec Family Study. Int J Obesity 16: 831-844

22. Province MA, Rao DC (1995) A general purpose model and a computer program for combined segregation and path analysis (SEGPATH): automatically creating 
computer programs from symbolic language model specifications. Genet Epidemiol 12: 203-219

23. Mitchell BD, Kammerer CM, Mahaney MC et al. (1996) Genetic analysis of the IRS: pleiotropic effects of genes influencing insulin levels on lipoproteins and obesity measures. Arterioscler Thromb Vasc Biol 16: 281-288

24. Mayer EJ, Newman B, Austin MA, et al. (1996) Genetic and environmental influences on insulin levels and the insulin resistance syndrome: an analysis of women twins. Am J Epidemiol 143: 323-332

25. Fain PR (1978) Characteristics of simple sibship variance tests for the detection of major loci and application to height, weight and spatial performance. Ann Hum Genet London 42: 109-120

26. Rao DC, MacLean CJ, Morton NE, Yee S (1975) Analysis of family resemblance. V. Height and weight in Northeastern Brazil. Am J Hum Genet 24: 509-520

27. Zonta LA, Jayakar SD, Bosisio M, Galante A, Pennetti V (1987) Genetic analysis of human obesity in an Italian sample. Hum Hered 37: 129-139

28. Karlin S, Williams PT, Jensen S, Farquhar JW (1981) Genetic analysis of the Stanford LRC Family Study data: I Structured exploratory data analysis of height and weight measurements. Am J Epidemiol 113: 307-324

29. Moll PP, Burns TL, Lauer RM (1991) The genetic and environmental sources of body mass index variability: the Muscatine Ponderosity Family Study. Am J Hum Genet 49: 1243-1255

30. Price RA, Ness R, Laskarzewski P (1990) Common major gene inheritance of extreme overweight. Hum Biol 62: 747-765

31. Province MA, Arnqvist P, Keller J, Higgins M, Rao DC (1990) Strong evidence for a major gene for obesity in the large, unselected total Community Health Study of Tecumseh. Am J Hum Genet 47 [Suppl]:A143 (Abstract)

32. Borecki IB, Bonney GE, Rice T, Bouchard C, Rao DC (1993) Influence of genotype-dependent effects of covariates on the outcome of segregation analysis of the body mass index. Am J Hum Genet 53: 676-687

33. Tiret L, André JL, Ducietière P et al. (1992) Segregation analysis of height-adjusted weight with generation- and age-dependent effects: the Nancy Family Study. Genet Epidemiol 9: 389-403
Appendix 1. Bivarate familial correlation model

\begin{tabular}{lllll}
\hline Group & $\mathrm{F}$ & $\mathrm{M}$ & $\mathrm{S}$ & $\mathrm{D}$ \\
\hline $\mathrm{F}$ & $\mathrm{R}_{\mathrm{F}}$ & $\mathrm{FM}$ & $\mathrm{FS}$ & $\mathrm{FD}$ \\
$\mathrm{M}$ & & $\mathrm{R}_{\mathrm{M}}$ & $\mathrm{MS}$ & $\mathrm{MD}$ \\
$\mathrm{S}$ & & & $\mathrm{RS}+\mathrm{SS}$ & $\mathrm{SD}$ \\
$\mathrm{D}$ & & & & $\mathrm{R}_{\mathrm{D}}+\mathrm{DD}$ \\
\hline
\end{tabular}

Note: Using element notation the matrices are defined as follows, where the subscript 1 denotes a body fat measure and subscript 2 reflects glucose, insulin, or IGR measures.

Interindividual-intergroup (full rank) matrices;

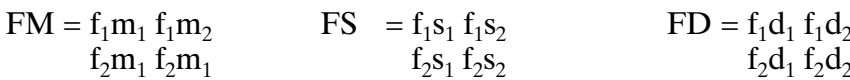

$$
\begin{aligned}
& \begin{array}{rrr}
\mathrm{MS}=\mathrm{m}_{1} \mathrm{~s}_{1} \mathrm{~m}_{1} \mathrm{~s}_{2} & \mathrm{MD}=\mathrm{m}_{1} \mathrm{~d}_{1} \mathrm{~m}_{1} \mathrm{~d}_{2} & \mathrm{SD}=\mathrm{s}_{1} \mathrm{~d}_{1} \mathrm{~s}_{1} \mathrm{~d}_{2} \\
\mathrm{~m}_{2} \mathrm{~s}_{1} \mathrm{~m}_{2} \mathrm{~s}_{2} & \mathrm{~m}_{2} \mathrm{~d}_{1} \mathrm{~m}_{2} \mathrm{~d}_{2} & \mathrm{~s}_{2} \mathrm{~d}_{1} \mathrm{~s}_{2} \mathrm{~s}_{2}
\end{array} \\
& \text { Interindividual-intergroup } \quad \mathrm{SS}=\mathrm{s}_{1} \mathrm{~s}_{1} \mathrm{~s}_{1} \mathrm{~s}_{2} \quad \mathrm{DD}=\mathrm{d}_{1} \mathrm{~d}_{1} \mathrm{~d}_{1} \mathrm{~d}_{2} \\
& \begin{array}{lll}
\text { (diagonal) matrices: } & \mathrm{s}_{2} \mathrm{~s}_{2} & \mathrm{~d}_{2} \mathrm{~d}_{2}
\end{array} \\
& \mathrm{R}_{\mathrm{F}}=1 \mathrm{f}_{12} \quad \mathrm{R}_{\mathrm{M}}=1 \mathrm{~m}_{12} \quad \mathrm{R}_{\mathrm{S}}=1 \mathrm{~s}_{12} \quad \mathrm{R}_{\mathrm{D}}=1 \mathrm{~d}_{12}
\end{aligned}
$$

F, Father; M, mother; S, son; D, daughter

The blank elements (lower off-diagonals in the latter six matrices) are equated with their respective upper off-diagonal elements (e.g., $s_{2} s_{1}=s_{1} s_{2}$ ). The total number of correlations estimated in the most general model is 34 . However, the number of cross-trait correlations (all off-diagonal elements) is 18, and constitutes the primary focus of this study 